\title{
LA “CAPACIDAD DE PERCEPCIÓN” DE LOS ADULTOS MAYORES EN LA DEFENSA DE SUS DERECHOS FUNDAMENTALES*
}

\author{
THE "PERCEPTION CAPABILITY” OF ELDERLY \\ PEOPLE IN THE DEFENSE OF THEIR FUNDAMENTAL \\ RIGHTS
}

A “CAPACIDADE DE PERCEPÇÃO” DO ADULTO MAIS VELHO NA DEFEŚA DOS DIREITOS

HUMANOS FUNDAMENTAIS

\section{RESUMEN}

Se parte del postulado básico reconocido por la legislación, la doctrina y la jurisprudencia internacional de que si bien los adultos mayores tienen las mismas prerrogativas que cualquier adulto, también lo es que su capacidad psíquica, social, emocional y física está disminuida, por lo que el sistema jurídico debe tratarlos en forma específica para lograr la efectividad de sus derechos fundamentales. La propuesta es que el propio sistema jurídico reconozca diferentes categorías de adultos mayores y que, en consecuencia, el régimen jurídico para cada categoría sea específico. Se sugiere que esa distinción parta de aceptar el concepto: capacidad de percepción. Concepto que puede incorporarse en la normativa de forma objetiva, subjetiva o mixta. La primera pretendería separar

*Artículo de investigación que presenta parte de los resultados de un proyecto de investigación realizado por los autores y financiado por el Gobierno Mexicano en la Facultad de Derecho y Ciencias Sociales de la Universidad Autónoma del Estado de Morelos.

a. Profesor investigador de tiempo completo en la Facultad de Derecho de la Universidad Autónoma del Estado de Morelos, México; profesor de la División de Estudios de Posgrado en Derecho de la UNAM; doctor en Derecho por la UNAM; doctor en Derecho por la Universidad Complutense de Madrid, España.

b. Profesora investigadora de tiempo completo en la Facultad de Derecho de la Universidad Autónoma del Estado de Morelos, México; doctora en Derecho por la Universidad de Viena, Austria y posgraduada en Derecho y Globalización por la Universidad de Castilla La Mancha, en Toledo, España.

Juan Manuel Ortega Maldonado juan.mom@hotmail.com Gabriela Mendizábal Bermúdez gabymendizabal@yahoo.com.mx
Fecha de recepción: 9 de Septiembre 2014 Fecha de revisión: 11 de Septiembre 2014 Fecha de aceptación: 24 de Noviembre 2014
MISIÓN JURÍDICA Revista de Derecho y Ciencias Sociales Bogotá, D.C. (Colombia)

Colaboradores Externos Internacionales Núm. 8, Año 2015

enero-junio, pp. 39-54

ISSN 1794-600X 
a los adultos mayores simplemente de acuerdo a su edad (como actualmente lo reconoce la legislación de muchos países); la segunda, por el contrario, los distinguiría conforme a criterios de apreciación sobre esa "capacidad de percepción" y la tercera, postula una diferenciación utilizando una combinación matizada de las dos posturas anteriores.

\section{PALABRAS CLAVE}

Capacidad de percepción, adultos mayores, legislación, edad biológica, derechos fundamentales y sociales.

\begin{abstract}
The authors start from the basic premise recognized by Law, doctrine and international jurisprudence, while older adults have the same rights as any adult, they also have their mental, social and physical abilities diminished, thus the legal system should treat them in a specific way for the realization of their Fundamental Rights. What authors propose is that the legal system itself should recognize different categories of older adults and that the laws should be specific for each category. The authors suggest these differential categories to be developed based on the concept of "perception capability". This concept can be incorporated into the regulations in any of the following ways: objective, subjective and mixed. The first would seek to separate the elderly according to their age (as the current legislation in many countries does); the second, by contrast, distinguishes the elderly according to assessment criteria based on perception capability and the third proposes a differentiation using a nuanced combination of the above mentioned two approaches.
\end{abstract}

\section{KEY-WORDS}

Perception capability, older adult, elderly.

\section{RESUMO}

Levando em conta o postulado básico reconhecido pela legislação, a doutrina e a jurisprudência internacional de que os adultos mais velhos têm as mesmas prerrogativas que qualquer adulto, também é certo que a sua capacidade social, emocional e físico-mental é diminuída; portanto o sistema jurídico deve tratá-los especificamente para alcançar a realização dos seus direitos fundamentais. A proposta é que o próprio sistema jurídico tem de reconhecer diferentes categorias de adultos mais velhos e que, por conseguinte, o regime jurídico para cada categoria seja específico. Sugere-se que esta distinção baseie-se em aceitar o conceito: "capacidade de percepção". Conceito que pode ser incorporado aos regulamentos de forma objetiva, subjetiva ou mista. A primeira buscaria separar os idosos simplesmente de acordo com sua idade (como atualmente reconhece a legislação de muitos países); a segunda, por outro lado, iria distingui-los de acordo com os critérios de apreciação sobre a "capacidade de percepção", e o terceiro postula uma diferenciação sutil, usando uma combinação das duas posições anteriores.

\section{PALAVRAS-CHAVE}

Capacidade de percepção, adultos mais velhos.

\section{INTRODUCCIÓN}

En la mayoría de los ordenamientos jurídicos latinoamericanos los adultos mayores son titulares de idénticos derechos constitucionales como cualquier otro ciudadano. No obstante ello, algunos adultos mayores poseen en muchos casos una capacidad psíquica y física en franco deterioro, lo cual reduce sus posibilidades de ejercer y disfrutar en plenitud de todos esos derechos.

De esta afirmación podemos derivar dos puntos de partida. Por un lado, hay que contar con que los adultos mayores no pueden ejercer desde el momento en que empiezan a serlo algunos derechos fundamentales de la misma manera en que podían hacerlo antes, especialmente aquéllos que requieren cierta manifestación física (como el derecho de expresión en manifestaciones públicas) o el derecho a la administración de justicia (que exige fortaleza moral). Se trata de una capacidad que se va deteriorando con el proceso paulatino del envejecimiento. Por otra parte, es un hecho irrefutable que los adultos mayores son más vulnerables y, por lo mismo, requieren mayor protección.

De estas reflexiones cabe, a su vez, inferir dos aseveraciones básicas. La primera, el alcance del ejercicio de los derechos fundamentales por los adultos mayores debe ser precisado en atención a 
sus características específicas. En este contexto es esencial no olvidar que tales matizaciones podrían hacer peligrar la universalidad de los derechos y libertades, por lo que habrá que ser sumamente cautos en este terreno.

Asimismo, en segundo lugar, es preciso considerar que los adultos mayores requieren un especial cuidado y protección de sus familiares, del Estado y de la sociedad en atención a su vulnerabilidad.

En tales condiciones, el objeto de este trabajo consiste en una aproximación a cómo se enfrenta -y cómo debería enfrentarse- nuestro ordenamiento a estos dos problemas. En el ámbito regulatorio se ha emprendido en México una importante reforma legislativa en materia de adultos mayores desde hace algunos años que nos permitirá reflexionar un poco más.

Sin embargo, se hace necesaria una aproximación constitucional ya que ésta es omisa hasta ahora en dispensar expresamente derechos especiales a los adultos mayores. Además, el modelo constitucional federal posee un especial interés para los ordenamientos estatales $y$ municipales.

A esto se suma el hecho de que el paulatino proceso de integración jurídica latinoamericana, a través de la Convención Americana de Derechos Humanos y de los pronunciamientos de la Corte Interamericana impone el conocimiento, o por lo menos la aproximación, al marco normativo en materia de derechos humanos del continente. No olvidemos que la Constitución Política mexicana fue reformada recientemente $y$, con ello, se ha impuesto un "nuevo sistema jurídico", en el que los Derechos Humanos tienen un rol primordial.

\section{PROBLEMA DE INVESTIGACIÓN}

Los adultos mayores en México son tratados jurídicamente en forma igual; sin embargo, este trato igualitario lo único que origina en realidad es un trato discriminatorio para aquellos adultos mayores que sufren una severa disminución de sus facultades físicas, psíquicas, emocionales y sociales con relación a otros adultos mayores que no tienen estos problemas. Conviene descartar aquellas situaciones en las que los adultos mayores sufren de una discapacidad objetiva o que han sido declarados incapaces. Nos referimos a aquellos adultos mayores que la legislación los declara aptos. Un adulto mayor no tiene las mismas capacidades a los 60 años que a los 90 años, ni tampoco las tiene un adulto mayor indígena que otro que no tiene esa condición. Pero a pesar de ello el sistema jurídico los trata en forma igualitaria. Por ello es dable cuestionarse ¿Debe el régimen jurídico de cada país hacer una distinción en cuanto al trato que le da a los adultos mayores, en relación a su capacidad de percepción y la protección de sus derechos fundamentales?

Dicho de otro modo, cómo lograr que
la normatividad mexicana considere
específicamente a los adultos mayores
disminuidos considerablemente de sus
capacidades físicas y psíquicas, quienes no tienen
representante legal para ejercer sus derechos
fundamentales, para que no estén en desventaja
con los adultos mayores en plenitud.

\section{METODOLOGÍA}

Para desarrollar el trabajo en un primer momento se utilizó el método analítico, pues se partió de estudiar los actuales enfoques del principio de igualdad y no discriminación que regula el sistema jurídico; seguidamente se utilizó el método comparativo para acreditar que existen diferencias emocionales, físicas, psíquicas y sociales entre los adultos mayores, distinciones que han sido ya puestas de relieve por otras disciplinas científicas. Posteriormente describiremos el concepto "capacidad de percepción" y la necesidad de su reconocimiento por el sistema jurídico.

Enseguida se postula que la normatividad está obligada a reconocer estas diferencias y a crear categorías jurídicas distintas y para ello utilizamos el método analógico con el propósito de obtener conclusiones similares a las que el sistema jurídico estableció para distinguir entre menores de edad, entre personas con alguna discapacidad, entre la población indígena y la que no lo es y entre mujeres y hombres. Finalmente, se establece que este concepto resultaría de primera importancia tanto para los tribunales como para las autoridades administrativas en la efectiva defensa de los derechos fundamentales de los adultos mayores. 


\section{EL PRINCIPIO DE IGUALDAD Y NO DISCRIMINACIÓN FRENTE A LOS ADULTOS MAYORES}

Si bien es cierto que estos conceptos están estrechamente vinculados, también lo es que no son idénticos, aunque sí complementarios. La idea de que no deben establecerse, ni permitirse distinciones entre los derechos de las personas con base en su nacionalidad, raza, sexo, religión o cualquier otra condición o circunstancia personal o social es consecuencia de que todas las personas son iguales; es decir, la noción de igualdad deriva directamente de la unidad de naturaleza del género humano y es inseparable de la dignidad de la persona, frente a la cual es incompatible toda situación que, por considerar superior a un determinado grupo, conduzca a tratarlo con privilegio o que, a la inversa, por considerarlo inferior, lo trate con hostilidad o de cualquier forma lo discrimine del goce de derechos que se reconocen a quienes no se consideran en tal situación de inferioridad. Así pues, no es admisible crear diferencias de trato entre seres humanos que no correspondan con su única e idéntica naturaleza.

Sin embargo, como la igualdad y la no discriminación se desprenden de la idea de unidad de dignidad y naturaleza de la persona, no todo tratamiento jurídico diferente es discriminatorio, porque no toda distinción de trato puede considerarse ofensiva, por sí misma, de la dignidad humana. Por tanto, la igualdad prevista por el artículo 4 de la Constitución Política de los Estados Unidos Mexicanos y en diversos instrumentos internacionales en materia de derechos humanos, más que un concepto de igualdad ordena al legislador no introducir distinciones entre ambos géneros $y$, si lo hace, éstas deben ser razonables y justificables.

El Máximo Tribunal mexicano, en pronunciamientos muy recientes, se ha venido refiriendo a este postulado con la denominación de "discriminación positiva o acción afirmativa"1. De esta forma ha establecido que la discriminación puede operar de manera legal o de hecho, por objeto o resultado (directa o indirecta), o a través de la omisión de adoptar medidas temporales diferenciadas para responder o evitar perpetuar situaciones de discriminación estructural.
Además, la discriminación puede tener un efecto único en el tiempo o puede operar también de manera continuada.

Si bien es cierto que el verdadero sentido de la igualdad es colocar a los particulares en condiciones de poder acceder a derechos reconocidos constitucionalmente, lo que implica eliminar situaciones de desigualdad manifiesta, ello no significa que todos los individuos deban ser iguales en todo, puesto que si la propia Constitución protege la propiedad privada, la libertad económica y otros derechos patrimoniales, está aceptando implícitamente la existencia de desigualdades materiales y económicas; es decir, el principio de igualdad no implica que todos los sujetos de la norma estén en todo momento y ante cualquier circunstancia, en condiciones de absoluta igualdad, sino que dicho principio se refiere a la igualdad jurídica, que debe traducirse en la seguridad de no tener que soportar un perjuicio (o privarse de un beneficio) desigual e injustificado.

En estas condiciones, el valor superior que persigue este principio consiste en evitar que existan normas que, llamadas a proyectarse sobre situaciones de igualdad de hecho, produzcan como efecto de su aplicación la ruptura de esa igualdad al generar un trato discriminatorio entre situaciones análogas, o bien, propicien efectos semejantes sobre personas que se están en situaciones dispares, lo que se traduce en desigualdad jurídica.

Dicho de otra manera, el postulado de igualdad que recoge la Constitución no exige que en México todos debamos ser iguales, al estilo que proponían las teorías comunistas más radicales. La misma Carta Fundamental reconoce diferencias materiales y económicas entre los mexicanos; pero lo que sí reconoce es la aspiración de todos a las mismas oportunidades económicas, culturales, educativas, sociales, políticas, etcétera.

Ahora bien, es verdad que el legislador posee libertad de configuración para regular la situación del adulto mayor, dicha facultad está limitada por los mandatos constitucionales y el reconocimiento de derechos humanos desde la Constitución y los tratados internacionales suscritos por México. 
Por eso, como lo anota la Suprema Corte:

“(...) el principio de igualdad y no discriminación aplica de manera transversal a los demás derechos humanos, y cualquier distinción, restricción, exclusión o preferencia en el ejercicio de dicho derecho que, además, se encuentre basada en alguna de las categorías prohibidas, constituye una violación del derecho citado...”2.

Así pues, tenemos que apuntar que existe un principio general de igualdad contenido en la Constitución Federal. Ésta alude en diversos artículos a este principio, por lo que en realidad, si bien se observa, la propia Constitución no es sino un listado de propósitos de igualdad, los cuales pueden analizarse desde tres perspectivas diferentes ${ }^{3}$.

1. Igualdad en la ley. La ley no debe establecer desigualdades, es decir, debe tratar igual a los iguales y desigual a los desiguales. Este es el propósito de los artículos 1ํㅜ 4ํㅜ , 12, 31, fracción IV, 132, 17 y 28 de la Norma Fundamental. El primero de ellos indica:

"Queda prohibida toda discriminación motivada por origen étnico o nacional, el género, la edad, las discapacidades, la condición social, las condiciones de salud, la religión, las opiniones, las preferencias, el estado civil o cualquier otra que atente contra la dignidad humana y tenga por objeto anular o menoscabar los derechos y libertades de las personas".

2. Igualdad por la ley. En este sentido la ley debe ser observada como instrumento para lograr la igualdad de las personas, corrigiendo las desigualdades económicas, políticas, sociales o de cualquier otra naturaleza, que imperan en un espacio y lugar determinado. A través de esta igualdad se busca la justicia social. En esta línea cabe señalar que este principio tiene también como propósito remover los obstáculos para que todos tengan el pleno desenvolvimiento de su personalidad y la protección de su dignidad, lo que significa generar un derecho social que proteja en forma sistemática a los menos favorecidos.

2 Tesis: 1a. CCLVIII/2014 (10a.) de fecha 04 de julio de 2014. 3 ORTEGA MALDONADO, Juan. Lecciones de Derecho Fiscal; Editorial Porrúa, México; 2012, p. 158.
La Constitución Federal busca este propósito a través de los artículos $2^{\circ}, 3^{\circ}, 4^{\circ}, 25,27,28,123$, etcétera. El profesor MoRENo PADILla escribe: "El Estado debe ser impulsor de las estructuras sociales, para que a través del derecho, toda la sociedad participe del cambio. Se trata de la igualdad como transformación social"4.

3. Igualdad ante la ley. Significa que la norma debe ser aplicada con criterio de estricta igualdad, a todos los afectados por ella. Aplicación que permite al operador jurídico valorar la "capacidad de percepción" del adulto mayor.

\section{ETAPAS BIOLÓGICAS, PSICOLÓGICAS Y SOCIALES ENTRE LOS ADULTOS MAYORES}

El estudio del envejecimiento humano se ha realizado por diversas ciencias (entre ellas la biología, la piscología y la sociología, como ahora veremos) que pretenden explicar el origen, las características y las fases que presenta. Ello, por un lado, ha enriquecido el análisis del fenómeno, pero, por otro, lo ha hecho más complejo pues no existe coincidencia en los conceptos ni en la metodología aplicados.

Lo cierto es que el proceso de envejecimiento es afectado por varios factores. Como bien lo apunta Berenice Bracho, “...envejecer es un proceso multifactorial (integrado por cambios endógenos y exógenos) que impone la indefectible pérdida o limitación de las capacidades y habilidades del individuo..."

\subsection{Edad biológica}

Se refiere a la edad cronológica y "se ha asociado a los efectos de las variables que tiene consecuencias tanto madurativas como degenerativas en el cuerpo humano"6. Según este criterio, la vejez se define a partir de los 60 o 65 años. "Desde esta perspectiva, el envejecimiento lleva consigo cambios en la posición del sujeto en la sociedad, debido a que muchas responsabilidades

4 MORENO PADILLA, Javier; El Principio de Igualdad en el Derecho Tributario; en la obra Principios Tributarios Constitucionales; TFF; México; 1989. p. 279.

5 HERNÁNDEZ BRACHO, Amalia. "Protección a las personas adultas mayores y en situación de dependencia en Morelos"; Tesis Doctoral, defendida el 30 de junio de 2014 en la Facultad de Derecho de la Universidad Autónoma del Estado de Morelos; p. 13 y 24.

6 VEGA VEGA, José y BUENO MARTÍNEZ, María. Desarrollo Adulto y envejecimiento; Ed. Síntesis, Madrid, 1999, p. 36. 
y privilegios sobre todo aquellos asociados al empleo, dependen de la edad cronológica"7.

En atención a este criterio, en la Unión Europea se aplica, para efectos legales y de servicios sociales, una división de las personas adultas mayores como de la tercera y cuarta edad.

La tercera edad inicia a los 65 años y finaliza a los 80 , en este grupo identifican a las personas que se encuentran relativamente saludables que pueden o no haberse retirado de la actividad laboral y buscan, ante esa desocupación, usar el tiempo libre con sentido, también los denominan viejos jóvenes.

La cuarta edad se extiende desde los 80 años en adelante y la han subdividido a su vez en dos grupos más que lo integran del modo siguiente:

a) Por el grupo integrado por los viejosviejos, personas de 80 a 95 años de edad, en el que se integran algunas personas activas, aunque la mayor parte requiere servicios sociales y sanitarios de apoyo, así como ayuda económica.

b) Los muy viejos y los centenarios, es el grupo integrado por personas que rebasan los 95 y 100 años de edad y que por el deterioro físico seguramente requieren servicios sociales, sanitarios y ayuda, en función del grado de dependencia de otras personas para sobrevivir.

Paula Aranibar señala que si se estudia la vejez a partir de la edad como elemento orientador es posible reconocer que en la medida en que "aumenta el grado de modernización de las sociedades, disminuye la valoración social de la vejez"8.

\subsection{Edad psicológica}

Estudiar la edad psicológica de los adultos mayores implica el análisis de las "capacidades del

7 HUENCHUAN, Sandra y RODRÍGUEZ PIÑERO, Luis; Envejecimiento y derechos humanos: situación y perspectivas de protección, CEPAL, ONU, Santiago de Chile, 2010, p. 14.

8 ARANIBAR, Paula; Acercamiento conceptual a la situación del adulto mayor en América Latina; Serie Población y Desarrollo, Centro Latinoamericano y Caribeño de Demografía, División de Población de la CEPAL, Área de población y desarrollo de la CELADE, Chile, 2001, p. 13. individuo para una conducta adaptativa" ${ }^{\prime \prime}$, implica actitudes y aptitudes respecto al proceso de envejecimiento en el que participa la percepción subjetiva de la persona, toda vez que envejecer es una experiencia individual que involucra no sólo el "recuerdo del pasado, también exige de la persona la capacidad de orientarse rápidamente en el presente utilizando la experiencia personal y ajena, de hecho irrepetible, así como los conocimientos adquiridos"10 para estar en condición de adaptarse al entorno en constante cambio.

Por eso es válido afirmar junto a Vega y Bueno que “...no hay una edad psicológica única sino tantas como ambientes concretos tengan que afrontar las personas..."11.

La edad psicológica tiene íntima relación con la edad cronológica en el sentido que al presentarse cambios y disminución en diferentes capacidades como la audición o la visión, esto puede suponer alteraciones o dificultades para una adecuada percepción del mundo que rodea al adulto mayor. Por ejemplo, en el caso específico de la pérdida progresiva de la capacidad auditiva, la persona queda poco a poco aislada de su entorno, originando un retraimiento $y$ una soledad involuntaria.

También se presentan cambios en la capacidad motriz, hay mayor lentitud para el desplazamiento y aparecen enfermedades crónico degenerativas como la artrosis; lo anterior, en conjunto, va a derivar en una disminución de la autonomía funcional de la persona y por tanto en una cada vez mayor dependencia de otras personas.

Es claro que estos casos pueden derivar en un impacto o afectación en el área psicológica del individuo; la mente de una persona adulta mayor puede encontrarse en perfectas condiciones de salud, sin embrago, la creciente dependencia de alguien más para moverse o para conectarse con su entorno ante una visión o audición disminuida, podrían impactar en la autoestima del sujeto.

Pero no sólo eso, sino también puede conducir a una desvinculación del individuo que

9 KRASSOIEVITCH, Miguel; Psicoterapia Geriátrica, Colección de Psicología, Fondo de Cultura Económica, México, 1993, p. 23. 10 Ibídem. Pág. 12

11 VEGA, José Luis, Bueno, Belén; op. cit., p. 43. 
se caracteriza por un alejamiento mutuo en el que la persona mayor reduce voluntariamente sus actividades y compromisos. A esta de por sí difícil situación se adiciona el hecho de que la sociedad estimula la segregación generacional presionando, entre otras cosas, para que la gente mayor se retire del mercado laboral y de la vida en sociedad.

Esta noción atiende especialmente a la percepción no sólo que el individuo tiene de sí mismo como persona frente a su proceso de envejecimiento, sino también ante los prejuicios de su entorno social que lo identifican como viejo, imponiendo cambios en su personalidad, en las metas que se traza y en las oportunidades a las que puede tener acceso.

\subsection{Edad social}

La edad social puede bien conceptualizarse como:

(...) una construcción que incluye a un conjunto de valores y símbolos culturales que la sociedad define y determina lo que considera el deber ser de las personas adultas mayores, es decir, son las expectativas que tiene su entorno social, para con los miembros de su grupo etario ${ }^{12}$.

De lo expuesto se advierte que la vejez no tiene únicamente un sentido biológico, sino también el significado que el grupo social le asigna a ser viejo, lo que influye en la percepción subjetiva de la persona adulta mayor, estableciendo las actitudes y conductas que se consideran adecuadas para una determina edad.

Además, la sociología ha hecho aportaciones importantes para la explicación de la edad social de los adultos mayores. Es así como se revela: a) la noción o tesis funcionalista sobre el envejecimiento, b) la noción de la economía política del envejecimiento y c) la teoría de la dependencia estructurada.

La primera considera a la vejez como "una forma de ruptura social $y$, en términos de la teoría del rol, como una pérdida progresiva de funciones"13. Este enfoque ha dado como resultado, por lo menos en el plano de la

12 KRASSOIEVITCH, Miguel; op.cit. p. 23

13 Cfr. HUENCHUAN, Sandra, ob. cit., p. 19-20 ciencia jurídica, entre otras anomalías, que sólo se haya estudiado a la vejez como un grupo económicamente dependiente, una carga para los sistemas de seguridad social y de salud y al que sólo se le podía mirar a través del asistencialismo.

Respecto a la noción de economía política de la vejez, se ha propuesto como un factor de análisis fundamental para comprender la situación de los adultos mayores la "posición y la calidad de vida que el individuo guarda en el mercado de trabajo al momento de la jubilación"14, que parece obvio es un enfoque capitalista. Se ha criticado esta postura pues no resulta acertado sostener que a los adultos mayores se les coloque sólo en una categoría social caracterizada por la desvinculación del sistema productivo y la falta de tributación a la seguridad social.

Con relación a la noción de dependencia estructurada, esta tesis expresa que el problema radica en el individualismo en el que vivimos. Esta forma de vida desvincula al sujeto de la actividad económica y que al final de sus días por eso representa una carga para la población activa. Por eso propone que se debe cuidar el sistema social en general, es decir, el cuidado y responsabilidad ante las personas adultas mayores debe provenir de la sociedad en su conjunto, lo que evitará la exclusión de estos.

Como hemos visto en el presente recorrido, la edad de los adultos mayores puede ser abordada desde diversos puntos de vista. Ahora comprendemos que ser un adulto mayor no sólo se presenta cuando se cumplen 60 años, sino que también existe una edad psicológica y una edad social que pueden ser coincidentes o no. Que, además, existen distintas etapas de la vejez plenamente identificadas. Pese a la ausencia de una idea generalizadora, lo importante es que nos permiten acercarnos al fenómeno del envejecimiento desde la perspectiva jurídica.

En efecto, a través de las normas que un Estado integra en su ordenamiento nacional es posible analizar cómo se diseñan no sólo las políticas públicas sino, sobre todo, cuál es la forma en que se concibe el fenómeno y cómo se comprende y aplica al grupo de los adultos mayores.

14 Cita de HUENCHUAN, Sandra; ob. cit. p. 20 


\section{LA CUESTIÓN DE LA CAPACIDAD DE PERCEPCIÓN A EFECTOS DEL EJERCICIO DE LOS DERECHOS FUNDAMENTALES}

\subsection{Concepto y contenido de la capacidad de percepción}

Como ya se advirtió con antelación, según los artículos 1 y 4 de la Constitución Federal mexicana, todas las personas somos iguales ante la ley, pero este principio de igualdad no exige, sin embargo, un trato idéntico a todos.

El no discriminar significa a menudo tener en cuenta las peculiaridades que obstaculizan o impiden la igualdad material. Sería ilógico e injusto no considerar que los adultos mayores posean una capacidad física y psíquica distinta al resto de los demás adultos.

No cabe ninguna duda de que los adultos mayores, son desde ese momento, titulares de los derechos humanos contenidos en la Constitución $\mathrm{y}$ en los tratados internacionales de los que el Estado mexicano es parte. La diferencia reside en el ejercicio de los mismos. Nos enfrentamos, pues, a una situación que en nuestra opinión sólo puede ser abordada de manera eficaz a partir de nuevos conceptos jurídicos, sin desconocer que posiblemente pueda bordarse también a partir de mecanismos de ejecución del orden normativo, sin que por el momento podamos ocuparnos de estos últimos.

Por eso insistimos en que una mejor aproximación puede venir de la mano del concepto "percepción" que nos ofrece la psicología y para convertirlo al sistema jurídico bien lo podemos denominar como capacidad de percepción. Este concepto implicaría la capacidad de orientarse rápidamente en el presente utilizando la experiencia personal y ajena, para estar en posibilidad de adaptarse al entorno en constante cambio. Este enfoque se centra en la forma en que las personas adultas mayores responden a las condiciones que les toca vivir, buscando la solución a sus problemas, aceptando y asumiendo pérdidas inevitables de forma que puedan seguir sintiéndose satisfechos e interesados en su existencia. Capacidad de percepción que podría medirse de diversas formas, según veremos.
Por el momento interesa destacar que esa capacidad de percepción está estrechamente vinculada con el ejercicio de los derechos fundamentales o la dependencia de dicho ejercicio a una determinada edad. Esta precisión debe ser hecha en forma muy cautelosa a fin de no dañar la universalidad de los derechos fundamentales.

No debemos olvidar que los adultos mayores, a diferencia de los menores, han pasado de ser considerados sujetos de pleno derecho (antes de los 60 años) a sujetos de protección (a partir precisamente de su consideración de personas con una capacidad disminuida).

A pesar de su relevancia, los derechos humanos no están plenamente garantizados de manera efectiva para los adultos mayores. Ello debido a que tales derechos fueron diseñados por adultos y pensados para adultos, pero no por adultos mayores. Si reconocemos que los adultos mayores son personas diferentes a los adultos, en consecuencia sus derechos fundamentales debieran ajustarse a partir de las características específicas de este colectivo.

\subsection{Capacidad de percepción y sistema jurídico}

El desarrollo del adulto mayor se da a lo largo de etapas, como ya indicamos, cada una de las cuales se caracteriza por la pérdida gradual de habilidades cognitivas, facultades físicas y determinadas características emocionales. En suma, se va perdiendo esa capacidad de percepción. De esta forma, la pérdida de las habilidades se va presentando de manera progresiva, lo que supone que según la etapa en la que se encuentre el adulto mayor, le será posible llevar a cabo ciertas operaciones mentales y tendrá determinadas características emocionales, y le será imposible realizar o tener otras.

Las habilidades cognitivas, sus facultades físicas y las características emocionales son de carácter estructural, es decir, no están sujetas a la voluntad de la persona (no son modificables), sino que se encuentran asociadas a la disminución de su desarrollo. Al respecto, es importante tener presente que si bien el nivel de desarrollo determina las capacidades que un adulto mayor puede desplegar, no es lo mismo que la edad cronológica. Ésta no necesariamente 
es coincidente con el nivel de desarrollo, de ahí que no deba ser el criterio a partir del cual se determine el posible nivel de desarrollo de una persona adulta mayor.

Pues bien, las características específicas del adulto mayor impactan de forma significativa en la forma en que rinde su testimonio, en sus habilidades para comprender el lenguaje hablado, en su capacidad para elaborar un relato de manera coherente y ordenada, o para recordar los hechos de manera precisa y suficiente, en la manera como controlar las emociones para sobrellevar una situación que le provoca angustia, en su capacidad de mantener la atención y concentración durante una diligencia judicial, cierta actividad administrativa, entre otros aspectos.

De ahí la necesidad de impulsar medidas especiales necesarias para que la participación de los adultos mayores en la defensa de sus derechos fundamentales sea idónea, garantizando de esta manera su pleno desarrollo.

Adicionalmente, los adultos mayores son considerados un grupo en situación de vulnerabilidad, entendiendo por ello aquellas personas que por su particular condición, como es la edad, género, color, raza, origen étnico, idioma, religión, opiniones políticas o de cualquier tipo, origen nacional, posición económica o condición social se encuentran en riesgo de que se vulneren sus derechos humanos.

Para el caso de los adultos mayores, el principal factor de vulnerabilidad es su edad, lo que no impide que además de ésta, tengan características adicionales que los coloquen en una situación de vulnerabilidad agravada, por ser adicionalmente mujer, indígena, tener una discapacidad o ser migrante, etc.

El hecho de que los adultos mayores sean considerados como grupo vulnerable por el Derecho Internacional de los Derechos Humanos no implica de ninguna manera que deban ser tratados únicamente como objetos de protección por parte del Estado. Por el contrario, deben volver a verse también como sujetos plenos de derechos.

Que los adultos mayores sean un grupo vulnerable conlleva para el Estado el impulso de medidas de protección especiales que les permitan el ejercicio de sus derechos. En otras palabras, la obligación del Estado de cara a los derechos del adulto mayor demanda también de una obligación reforzada para que esos derechos puedan concretarse en la realidad.

Por lo anterior, el Estado tiene el deber de garantizar los derechos de los adultos con una serie de medidas especiales, lo cual también supone la adopción de una serie de medidas especiales que permitan que los adultos mayores puedan verdaderamente ejercer estos derechos.

\subsection{La capacidad de percepción en las distintas manifestaciones de envejecimiento}

Este enfoque, de cualquier forma, debe tomar en cuenta tres nociones fundamentales en los adultos mayores, como bien lo anota Caro López ${ }^{15}$ : envejecimiento físico, envejecimiento biológico y envejecimiento social o cultural.

El envejecimiento físico está marcado por cambios en la apariencia física de las personas, entre ellos la pérdida de la elasticidad de la piel, adelgazamiento y decoloración del cabello, cambio en el tono de voz y aumento de las áreas de pigmentación, entre otros.

El biológico se manifiesta en niveles de trastorno o deterioro funcional y está íntimamente ligado al envejecimiento físico, ya que un número importante de los cambios que tienen lugar en los adultos mayores son resultado del deterioro de los sentidos y órganos, como disminución de la vista, de la capacidad auditiva, de la memoria o baja del rendimiento cardiaco.

El envejecimiento social o cultural corresponde a las actitudes y conductas sociales que se consideran adecuadas para una determinada edad; es decir, se refiere a la forma en cómo las culturas construyen la imagen de lo viejo y del anciano y a la asignación de roles y posiciones que establece la sociedad para los adultos mayores.

Existen múltiples factores externos como la alimentación, enfermedades, ejercicio, contaminación, condiciones de trabajo, etcétera, que contribuyen al retraso o aceleración de los

15 CARO LÓPEZ, Elizabeth; "Nuevas Políticas para Adultos Mayores: El caso del Distrito Federal. De la asistencia a la participación social; en Revista de Administración Pública, $N^{\circ}$ 109, p. 76. 
diferentes tipos de envejecimiento, por lo que éste se da en diferente grado y forma para cada persona.

Ello se afirma porque si de lo que se trata es de analizar las aptitudes de una persona adulta mayor para defender sus derechos fundamentales, entonces debemos convenir en que se hace necesario referirnos a la noción de "capacidad de percepción" para la defensa efectiva de los derechos humanos de los adultos mayores.

\subsection{Capacidad de percepción y derechos fundamentales}

Siguiendo este trazo, podemos distinguir entre la actuación jurídica y la actuación física o natural. En el campo de la actuación jurídica son los límites fijados legalmente los que determinan la capacidad de ejercicio. En este sentido cabe señalar los derechos fundamentales recogidos en los artículos 4 (protección constitucional del matrimonio y la familia), 14 y 27 (derecho a la propiedad privada y a heredar).

En cuanto a las actuaciones no reguladas formalmente, por el contrario, la capacidad para el ejercicio de los derechos fundamentales depende únicamente de las facultades psíquicas de cada persona. Es el caso de los derechos fundamentales recogidos en los artículos 3 (derecho a la educación), 6 (libertad de expresión), 8 (derecho de petición), 16 (derecho a la privacidad de las comunicaciones), y otros. Todos estos derechos mantienen una estrecha dependencia respecto del grado de percepción individual.

Por eso nos pronunciamos en contra del requisito de una edad determinada, ya que esta solución no toma en cuenta el progresivo deterioro de la personalidad del adulto mayor. No es lo mismo una persona de 60 años (que es la edad mínima para ser considerado adulto mayor), que una persona de 90 años. De la misma manera en que no puede afirmarse el mismo nivel de madurez intelectual y emocional de un niño de 8 años y uno de 16.

\subsection{Conflictos del adulto mayor con los poderes públicos y con sus familiares}

Ahora bien, pueden surgir conflictos en dos ámbitos: en la relación directa del adulto mayor respecto a los poderes públicos, circunstancia para la que la ley no contempla limitación alguna por motivos de disminución de facultades y en el derecho y obligación de los familiares al cuidado de los adultos mayores, que puede conducir de forma progresiva con el transcurso del tiempo a colisiones con el derecho al libre desarrollo de la personalidad, de la educación o de cualquier otro derecho del adulto mayor.

La relación de un adulto mayor con el Estado se desarrolla principalmente en el marco de instituciones públicas como el $\mathrm{DIF}^{16}$, pero aquella también se hace patente cuando el adulto mayor participa, por ejemplo, en una manifestación. Especialmente conflictivo puede ser la "capacidad de percepción" a efectos de los derechos fundamentales frente al derecho de los familiares de cuidado del adulto mayor. La constante e imparable disminución del grado de percepción de un adulto mayor, conlleva irremediablemente un correlativo aumento del derecho-obligación de sus familiares a su cuidado.

Que el bienestar del adulto mayor deba ser la pauta de decisión que trace la difícil frontera entre los derechos de aquéllos y de sus familiares, o que estos familiares tengan la obligación de ejercer todos aquellos aspectos de su deber de cuidado y protección desde el principio fundamental de respeto a la dignidad de la persona, son efectivamente criterios muy notables a considerar; que aunque no excluyen el conflicto, si muestran que tropezamos con los límites de la esfera privada de la familia.

Resumiendo esta primera parte del trabajo, podemos decir que los adultos mayores siguen siendo titulares o sujetos de los derechos fundamentales, pero no sería realista ignorar la progresiva reducción de sus aptitudes morales, intelectuales y físicas, pues estas tienen decisivas consecuencias para el ejercicio consciente de los derechos fundamentales. ¿Qué sentido real puede tener, por ejemplo, el derecho a la propiedad, si un individuo no tiene la capacidad de razonar con claridad sus alcances? A dar una respuesta a esta problemática debe contribuir el concepto

16 Se trata de las iniciales de una institución pública que significan literalmente Desarrollo Integral de la Familia, y que en realidad constituyen todo un Sistema con ese propósito a nivel nacional y local. 
de "capacidad de percepción" para efectos de los derechos fundamentales.

En los conflictos entre los adultos mayores respecto al Estado o a los familiares, sería tal vez lo más conveniente, a pesar de la dificultad de su aplicación práctica, atender al grado de capacidad de percepción de cada adulto mayor, jugando el bienestar del anciano y el principio de máxima libertad posible como criterios decisivos. Las obligaciones de los poderes públicos del Estado comprenden un especial deber de protección de los derechos de los adultos mayores.

No obstante la verdadera demostración de que un derecho está vigente y es efectivo la proporciona exclusivamente su garantía procesal. La posibilidad de invocarlo a los tribunales. Aquí también son aplicables los principios citados en el párrafo anterior. Los problemas más agudos se hallan, sin embargo, cuando a esa capacidad de ejercicio se le aúnan consecuencias económicas. En razón de la especial vulnerabilidad y necesidad de protección del adulto mayor, la legitimación para la interposición de los medios de defensa únicamente se debe regir por un principio de máxima autonomía mientras no le suponga un perjuicio eco.

\section{SOBRE LA NECESIDAD DE DIFERENCIAR ENTRE LOS ADULTOS MAYORES}

\subsection{Del reconocimiento de las desigualdades de hecho entre los adultos mayores}

La existencia de desigualdades de hecho en el ejercicio de cualquier derecho fundamental de los adultos mayores, impone la necesidad de hacer una distinción en el trato. Si bien pudiera cuestionarse argumentado la vulneración del principio de igualdad y no discriminación ${ }^{17}$, en

17 De acuerdo con la Corte Interamericana de Derechos Humanos la discriminación es toda exclusión, privilegio o restricción -que carece de objetividad y razonabilidad- que implica un detrimento de los derechos humanos. La igualdad, por su parte, es una característica esencial de la persona que se desprende de la dignidad humana, de acuerdo con la cual es incompatible considerar a un grupo como superior y tratarlo con privilegio o, a la inversa, considerarlo inferior y tratarlo con hostilidad. Cfr. Corte IDH. OC18/03 Condición Jurídica y derechos de los migrantes indocumentados. 17 de septiembre de 2003. Serie A No. 18, párr. 87. Corte IDH. Opinión Consultiva OC-17/02 Condición jurídica y derechos humanos del niño. 28 de agosto de tanto se trata de una distinción fundamentada en desigualdades reales, se vuelve indispensable para el ejercicio pleno estos derechos y para no transgredir el principio de igualdad y no discriminación.

Reconociendo que los adultos mayores, a partir de sus características propias de desarrollo cognitivo, emocional y social, no son iguales a otros adultos (los menores de 60 años) e incluso, entre otros adultos mayores (los mayores de 80 y 95 años), y que, en consecuencia, no pueden ejercer algunos derechos en las mismas condiciones que aquellos, el Estado se encuentra obligado a hacer una distinción de trato.

Debemos recordar, junto al Supremo Tribunal del país, que: “...el principio de igualdad debe entenderse como la exigencia constitucional de tratar igual a los iguales y desigual a los desiguales, de ahí que en algunas ocasiones hacer distinciones estará... permitido o, incluso, constitucionalmente exigido..."18.

Con el fin de cerrar este apartado diremos que para poder cumplir con este principio de igualdad y no discriminación, el legislador no sólo está facultado, sino que tiene obligación de crear categorías o clasificaciones de adultos mayores, a condición de que éstas no sean caprichosas o arbitrarias, o creadas para hostilizar a determinadas clases o universalidades de ciudadanos, esto es, que se sustenten en bases objetivas que justifiquen el tratamiento diferente entre una y otra categoría, y que pueden responder a finalidades económicas, psicológicas, biológicas, sociales o razones de política social.

Sostenemos que algunas de las directrices que pueden tomarse en cuenta para esa clasificación, serían las que más adelante se proponen.

\subsection{La igualdad en la Ley y los adultos mayores}

Este mecanismo de clasificación entre los adultos mayores al que nos referimos debe necesariamente aparecer en ley. Advertimos que la ley ordinaria (Ley del Adulto Mayor)

2002. Serie A No. 17, párr. 45.

18 Tesis de jurisprudencia 55/2006 emitida por la Primera Sala de la SCJN, Septiembre de2006., tomo XXIV, SJF. 
es irrespetuosa del principio de igualdad y no discriminación por agrupar a todos los adultos mayores después de los 60 sin hacer distinciones entre ellos, siendo que tales distinciones existen. Estimamos que esta ley debe reconocer una clasificación de los adultos, según su grado de vulnerabilidad.

Entendemos que legislar implica necesariamente clasificar y distinguir casos que en cualquier medida legal clasificatoria opera una afectación de expectativas o derechos. Sin embargo, cuando las autoridades se ven llamadas a actuar como garantes del principio de igualdad y no discriminación, ello las obliga a examinar rasgos adicionales a los que se consideran cuando se contempla la cuestión desde la perspectiva de los derechos sustantivos involucrados.

Así, aunque se haya concluido que una determinada regulación limitadora de derechos no es excesiva sino legítima, necesaria y proporcional, justificada por la necesidad de armonizar las exigencias normativas derivadas del derecho alegado por un adulto mayor, por ejemplo, todavía puede ser necesario analizar, bajo el principio de igualdad, si las cargas que esa limitación de derechos representa están repartidas utilizando criterios clasificatorios legítimos y suficientes.

Esto es, aunque una norma legal sea adecuada en el sentido de representar una medida globalmente apta para tratar de alcanzar un determinado fin, puede tener defectos de sobreinclusión o de infra-inclusión (como es el caso de los adultos mayores de estar todos incluidos en la misma categoría a partir de los 60), de los que derive una vulneración del principio de igualdad y no discriminación. La clasificación deberá entonces estar basada en la vulnerabilidad del individuo y no necesariamente en la edad.

Incluso, en algunas ocasiones, por el tipo de criterio usado por la Ley del Adulto Mayor (origen étnico o nacional, el género, la edad, las discapacidades, la condición social, las condiciones de salud, la religión, las opiniones, las preferencias, el estado civil o cualquier otra que atente contra la dignidad humana y tenga por objeto anular o menoscabar los derechos y libertades de las personas) o por la extensión e intensidad de la incidencia en el goce de un derecho fundamental, será necesario examinar con especial cuidado si los medios (distinciones inexistentes) usados por el legislador son adecuados y suficientes a la luz del fin perseguido.

\subsection{Sobre la intensidad en el escrutinio del principio de igualdad para los adultos mayores}

Por otro lado no podemos dejar de subrayar que la igualdad es un principio y un derecho de carácter fundamentalmente adjetivo que se predica siempre de algo, y éste referente es relevante al momento de realizar el control de constitucionalidad de las leyes, porque la Constitución permite que en algunos ámbitos el legislador tenga más amplitud para desarrollar su labor normativa, mientras que en otros se debe ser más exigente a la hora de determinar si aquél ha respetado las exigencias del principio de igualdad.

El artículo 1o. de la Constitución Federal establece varios casos en los que procede dicho escrutinio estricto. Así, su primer párrafo proclama que "todo individuo debe gozar de las garantías que ella otorga, las cuales no pueden restringirse ni suspenderse sino en los casos y con las condiciones que la misma establece", lo que evidencia la voluntad constitucional de asegurar en los más amplios términos el goce de los derechos fundamentales, y de que las limitaciones a ellos sean concebidas restrictivamente, de conformidad con el carácter excepcional que la Constitución les atribuye.

Por ello, siempre que la acción clasificadora del legislador incida en los derechos fundamentales garantizados constitucionalmente, será necesario aplicar con especial intensidad las exigencias derivadas del principio de igualdad y no discriminación.

Por su parte, el párrafo tercero del citado precepto constitucional muestra la voluntad de extender la garantía de igualdad a ámbitos que trascienden el campo delimitado por el respeto a los derechos fundamentales explícitamente otorgados por la Constitución, al prohibir al legislador que en el desarrollo general de su labor incurra en discriminación por una serie de motivos enumerados (origen étnico o nacional, género, edad, capacidades diferentes, condición social, condiciones de salud, religión, opiniones, 
preferencias, estado civil) o en cualquier otro que atente contra la dignidad humana y tenga por objeto anular o menoscabar los derechos y libertades de las personas.

La intención constitucional es, por lo tanto, extender las garantías implícitas en el principio de igualdad al ámbito de las acciones legislativas que tienen un impacto significativo en la libertad y la dignidad de las personas, así como al de aquellas que se articulan en torno al uso de una serie de criterios clasificatorios mencionados en el referido tercer párrafo, sin que ello implique que al legislador le esté vedado absolutamente el uso de dichas categorías en el desarrollo de su labor normativa, sino que debe ser especialmente cuidadoso al hacerlo.

En esos casos, se deberá someter la labor del legislador a un escrutinio especialmente cuidadoso desde el punto de vista del respeto a la garantía de igualdad.

En resumen, existen dos niveles de análisis de la constitucionalidad, uno de carácter ordinario y otro de nivel intenso. El primero debe realizarse en los asuntos que no incidan directamente sobre los derechos humanos y exista un amplio margen de acción y apreciación para la autoridad desde el punto de vista normativo, como ocurre en la materia económica o financiera. En cambio, el escrutinio estricto se actualiza cuando el caso que se tenga que resolver involucre categorías que no parecen tener cabida dentro de los términos del artículo 10 de la Constitución; se afecten derechos humanos reconocidos por el propio texto constitucional y/o por los tratados internacionales, o se incida directamente sobre la configuración legislativa que la Constitución prevé de manera específica para la actuación de las autoridades de los distintos niveles de gobierno.

\subsection{Propuesta de criterios de clasificación entre adultos mayores}

Ahora, nosotros postulamos que esa distinción puede darse, como ya se adelantó, utilizando el concepto de capacidad de percepción. Esta diferenciación implicaría que la normatividad dispense un trato más protector a aquellos adultos mayores cuya capacidad de percepción se encuentre disminuida. Entonces hablaríamos de una ecuación simple: entre menor sea la capacidad de percepción, mayor será la protección que debe otorgarse al adulto mayor. Eso permitiría tratar en forma distinta a un adulto mayor de 60 años que a otro de 80 o de 95.

Ahora bien, la utilización de este concepto tácitamente ha sido reconocida por la mayoría de las legislaciones porque todas coinciden en que se empieza a ser adulto mayor a partir de determinada edad (generalmente 60 o 65 años). Esto es, se reconoce que la capacidad de percepción empieza a disminuir precisamente en esa edad. Se trata de un dato objetivo.

En la Unión Europea se divide a los adultos mayores en dos grupos: un primer grupo que va de los 65 a los 80 y un segundo grupo que va de los 81 en adelante. Incluso habría la posibilidad de referirse también a adultos mayores de 95 años. Esa puede ser una propuesta interesante que permita a las autoridades contar con un dato objetivo para efectos de la aplicación de determinado régimen jurídico cada vez más favorable.

Por otro lado estaría la posibilidad de descartar totalmente esta postura y remitirse a una tesis subjetiva bajo la cual el operador jurídico podría valorar "libremente" la aplicación de determinado régimen más o menos favorable para un adulto mayor en función a su capacidad de percepción. Si estima que la capacidad de percepción del adulto mayor, cuya situación estaría analizando, se encuentra seriamente afectada, entonces podría dispensar una aplicación en extremo favorable para esa persona. Pongamos para efectos prácticos, como ejemplo, una absoluta suplencia de la queja deficiente en los juicios que llegue a tramitar. $\mathrm{Si}$, por el contrario, estima que su capacidad de percepción es plena, entonces no habría necesidad de aplicar más que los beneficios ordinarios para cualquier adulto mayor.

Sin embargo, no estamos de acuerdo con ninguna de las tesis anteriores. Antes bien, sostenemos que no resulta prudente colocarse en alguno de esos extremos, sino que el sistema jurídico debería atemperar el rigor con el que pudieran llegar a aplicarse tanto el sistema objetivo como el subjetivo.

Por eso creemos que sería necesario postular que una sana mezcla entre ambas tesis resulta saludable. Para ello entonces la legislación debería partir de un dato objetivo y dividir a los adultos 
mayores en tres grandes grupos como lo han hecho ya disciplinas como la biología, apoyada por la psicología y la sociología. De esta suerte tendríamos los siguientes regímenes jurídicos aplicables a los adultos mayores:

Primera etapa: 60 a 85 años

Segunda etapa: 85 a 95

Tercera etapa: 95 en adelante.

Algo muy importante que debe anotarse es que este dato objetivo sólo serviría como referencia, pero no constituiría ninguna camisa de fuerza para la aplicación de un específico régimen normativo. El operador tendría la posibilidad, apoyado por profesionales especializados, de aplicar un régimen más favorable (nunca menos favorable) cuando estime que el adulto mayor tiene una capacidad de percepción seriamente disminuida en relación al estándar o media de su edad.

En otras palabras se procuraría que la intensidad del escrutinio, a la luz de los principios de capacidad de percepción y plena autonomía del adulto mayor, no sean de carácter estricto, sino flexibles o laxos.

Reiterando diremos que cuando la acción del legislador incida en los derechos fundamentales garantizados por la Constitución, será necesario aplicar con especial intensidad las exigencias derivadas del principio de igualdad y no discriminación y si para ello es necesario crear clasificaciones de los sujetos beneficiados, el legislador estaría obligado a proceder en esa forma.

Por el contrario, en el caso de normatividad con efectos económicos o tributarios, por regla general, la intensidad del análisis constitucional debe ser poco estricta, con el fin de no vulnerar la libertad política del legislador, en campos como el económico, en donde la propia Constitución establece una amplia capacidad de intervención y regulación diferenciada del Estado, considerando que, cuando el texto constitucional establece un margen de discrecionalidad en ciertas materias, eso significa que las posibilidades de injerencia de las autoridades son menores $y$, por ende, la intensidad de su control se ve limitada.

En tales esferas, un control muy estricto llevaría al juez constitucional a sustituir la competencia legislativa del Congreso -o la extraordinaria que puede corresponder al Ejecutivo-, pues no es función del Poder Judicial Federal, sino de los órganos políticos, entrar a analizar si esas clasificaciones económicas son las mejores o si éstas resultan necesarias. Pero fuera de tales clasificaciones económicas, las clasificaciones sobre adultos mayores se imponen.

Esta solución resulta mucho más ajustada a los principios jurídicos relativos a los derechos humanos, amparados por la Constitución y los tratados internacionales en esta materia.

Cada rango normativo debería tener consecuencias jurídicas distintas tanto para efectos de prestaciones sociales y asistenciales, como familiares, civiles, penales, laborales, fiscales, administrativas, etcétera. Lo ideal sería contar con una regulación específica para este grupo vulnerable y sus propias excepciones ${ }^{19}$, pero ante esta imposibilidad lo mejor es que el operador jurídico atempere el rigor de las normas y obligaciones contenidas en todos los ordenamientos jurídicos para ser aplicados a los adultos mayores con mayor o menor rigor, como hemos anotado.

\section{RESULTADOS}

Como resultado de los grandes cambios que se han presentado en nuestro país respecto a las cuestiones relativas a la edad de los individuos,

19 Por ejemplo, en el artículo 284 del Código Civil para el Estado de Chiapas (vigente a partir del doce de septiembre de dos mil siete), en su tercer párrafo dispone que en el divorcio por mutuo consentimiento, los cónyuges no tienen derecho a pensión alimentaria, salvo pacto en contrario; asimismo, en el párrafo siguiente se prevé que tratándose del mismo tipo de divorcio y en aquellos casos en los que no exista cónyuge culpable, la mujer tendrá derecho de recibir alimentos por el mismo lapso de duración del matrimonio, derecho que disfrutará: a) si no tiene ingresos suficientes y b) mientras no contraiga nuevas nupcias $o$ se una en concubinato Las anteriores disposiciones no son contradictorias, pues el tercer párrafo prevé una regla general acorde con la igualdad formal entre cónyuges, mientras que el párrafo cuarto establece una excepción a favor de la mujer instituida por el legislador "con el objeto de dotar de equidad a los cónyuges", esto es, se trata de una acción afirmativa o medida legislativa de discriminación positiva, creada a partir del reconocimiento de que en nuestro país las tareas domésticas $y$ el cuidado de los hijos del matrimonio continúa siendo responsabilidad preponderante de la mujer, lo que provoca un estado de desigualdad material con el varón con motivo del divorcio; de ahí que dicha medida busca establecer una igualdad material de género, acción afirmativa que resulta acorde con los artículos 1o. y 4o., párrafo primero, de la Constitución Política de los Estados Unidos Mexicanos y con la Convención sobre la Eliminación de todas las Formas de Discriminación contra la Mujer (CEDAW por sus siglas en inglés). 
puesto que determinados estudios reflejan que más del 50\% de la población es joven y los adultos mayores sufren cambios significativos en su esfera personal y profesional, debido a que con el paso mismo de los años se presenta un notable deterioro físico, psíquico, económico, social y emocional, lo cual los imposibilita para ejercer sus derechos fundamentales de forma plena.

Si bien es cierto que nuestra legislación reconoce a las personas adultas mayores una igualdad para el acceso al trabajo, también es oportuno reconocer que esta situación no es totalmente cierta. Por ello y como resultado de las investigaciones realizadas, consideramos que las personas adultas mayores deberían ser tratadas por nuestro sistema jurídico de forma particular o diferenciada, tomando en consideración que los adultos mayores son vulnerables y por ende, requieren de mayor protección por parte del Estado

Se espera proponer a consideración de las instancias gubernativas las conclusiones del presente trabajo para el efecto de que puedan incorporarse a la ley en el nivel federal y en el estatal.

\section{CONCLUSIONES}

1. Cuando hablamos de la capacidad de los adultos mayores para proteger por sí mismos sus derechos fundamentales, no podemos soslayar su singular situación psíquica, emocional y física, ya que estas condiciones tienen decisivas consecuencias para el ejercicio consciente de los derechos fundamentales. A solucionar este problema debe contribuir el concepto de "capacidad de percepción".

2. El principio que ha de guiarnos ha de ser el de máximo posible de reconocimiento de capacidad a efectos de titularidad y ejercicio de los derechos fundamentales, a fin de garantizar la universalidad de los mismos. Cuando existan conflictos entre los derechos de los adultos mayores y el Estados y/o sus familiares, el concepto de capacidad de percepción vendría bien, combinándolo con el principio de bienestar del adulto mayor y el principio de máxima libertad posible como criterios decisivos.

3. Hemos constatado que la preocupación por el bienestar de los adultos mayores está efectivamente presente en el ordenamiento nacional. A pesar de que a nivel constitucional no encontramos una mención expresa a la especial necesidad de cuidado de los adultos mayores, los derechos fundamentales existentes son lo suficientemente amplios, detallados y universales para garantizar y dar cabida a una particular protección de este grupo vulnerable. Un papel clave desempeña en este sentido la posición central de la dignidad de la persona y el derecho al libre desarrollo de la personalidad (artículos 1 y 25).

4. Se pretende proteger a los adultos mayores en relación a su grado de vulnerabilidad física o psíquica y no en relación a su edad. 


\section{BIBLIOGRAFÍA}

- ARANIBAR, Paula; Acercamiento conceptual a la situación del adulto mayor en América Latina; Serie Población y Desarrollo, Centro Latinoamericano y Caribeño de Demografía, División de Población de la CEPAL, Área de población y desarrollo de la CELADE, Chile, 2001, 70 p.

- CARO LÓPEZ, Elizabeth; “Nuevas Políticas para Adultos Mayores: El caso del Distrito Federal. De la asistencia a la participación social; EN: Revista de Administración Pública, $N^{\circ} 109,12$ p.

- Convención Americana sobre Derechos Humanos, adoptada en la ciudad de San José de Costa Rica, el 22 de noviembre de 1969 Convención publicada en el diario oficial de la federación, el jueves 7 de mayo de 1981.

- Gaceta Parlamentaria, año IV, número 714, viernes 23 de marzo de 2001. Pro1puesta a cargo del diputado Pedro Miguel Rosalindo Salazar del PRD.

- Gaceta Parlamentaria, año V, número 1115 , miércoles 23 de octubre de 2002.

- Gaceta Parlamentaria de la Cámara de Diputados de fecha 18 de octubre de 2011.

- Hernández Bracho, Amalia Berenice; "Protección a las personas adultas mayores y en situación de dependencia en Morelos"; Tesis Doctoral, defendida el 30 de junio de 2014 en la Facultad de Derecho de la Universidad Autónoma del Estado de Morelos; p. 13 y 24.

- HUENCHUAN, Sandra y RODRÍGUEZ Piñero, Luis; Envejecimiento y derechos humanos: situación y perspectivas de protección, CEPAL, ONU, Santiago de Chile, 2010, 144 p.

- KRASSOIEVITCH, Miguel; Psicoterapia Geriátrica, Colección de Psicología, Fondo de Cultura Económica, México, 1993, 269 p.
- Ley de los Derechos de las Personas Adultas Mayores Publicada en el DOF de fecha 25 de junio de 2002.

- MORENO PADILLA, Javier; El Principio de Igualdad en el Derecho Tributario; en la obra Principios Tributarios Constitucionales; TFF; México; 1989. p. 279.

- ORTEGA MALDONADO, Juan Manuel; Lecciones de Derecho Fiscal; Editorial Porrúa, México; 2012, 328 p.

- Pacto Internacional de Derechos Civiles y Políticos DOF 20 de mayo de 1981.

- Pacto Internacional de Derechos Económicos, Sociales y Culturales DOF 12 de mayo de 1981.

- PRINCIPIOS DE LAS NACIONES UNIDAS EN FAVOR DE LAS PERSONAS DE EDAD (adoptó el 16 de diciembre de 1991 - Resolución 46/91).

- Protocolo Adicional a la Convención Americana Sobre Derechos Humanos.

- Protocolo de San Salvador Protocolo publicado en el Diario Oficial de la Federación, el martes 1 de septiembre de 1998.

- Tesis de jurisprudencia 55/2006 emitida por la Primera Sala de la SCJN, Septiembre de2006., tomo XXIV, SJF.

- Tesis: XXVII.10.(VIII Región) 3 C (10a.). Página: 2364. Registro electrónico: 2001828

- Tesis: 1a. CCLVIII/2014 (10a.) de fecha 04 de julio de 2014.

- VEGA, José Luis y BUENO MARTÍNEZ, María Belén; Desarrollo Adulto y envejecimiento; Ed. Síntesis, Madrid, 1999, 368 p. 